\title{
ROTURA GÁSTRICA POR BAROTRAUMA
}

\section{BAROGENIC RUPTURE OF THE STOMACH}

\author{
Rogerio Saad-Hossne, TCBC-SP ${ }^{1}$; Renê Gaberini Prado,TCBC-SP ${ }^{1}$; Alexandre Bakoniy Neto ${ }^{1}$; \\ Rodrigo Severo de Camargo Pereira²; Roberta Thiery Godoy Arashiro ${ }^{2}$
}

\section{INTRODUÇÃO}

A rotura gástrica por barotrauma constitui uma causa rara de abdome agudo perfurativo, tendo sido encontrados poucos relatos na literatura ${ }^{1-4}$. É geralmente tratada por laparotomia exploradora e rafia primária da lesão, porém, segundo Yeung et $a l^{1}$, em alguns casos é possível adotar-se conduta conservadora. Descrevemos um caso de rotura traumática de estômago pós intubação oroesofágica.

\section{RELATO DO CASO}

S. C. P., 20 anos, sexo feminino, puerpera, em tratamento de tuberculose pulmonar com isoniazida e rifampicina, apresentou no $5^{\circ}$ dia de puerpério, piora do quadro pulmonar, intensificação da dispnéia, tosse produtiva e febre; o diagnóstico clinico e radiológico foi compatível com broncopneumonia. Evoluiu com piora progressiva do quadro, mesmo em vigência de antibioticoterapia de amplo espectro (imipenem e clindamicina), progredindo para insuficiência respiratória aguda, necessitando de intubação orotraqueal e ventilação mecânica. Após a entubação, evoluiu com distensão abdominal importante, desaparecimento da macicez hepática (sinal de Jobert) e dificuldade de ventilação, sendo então constatada canulação iatrogênica do esôfago. Realizado exame radiográfico simples do abdome, que demonstrou pneumoperitônio extenso (Figura 1). Indicada

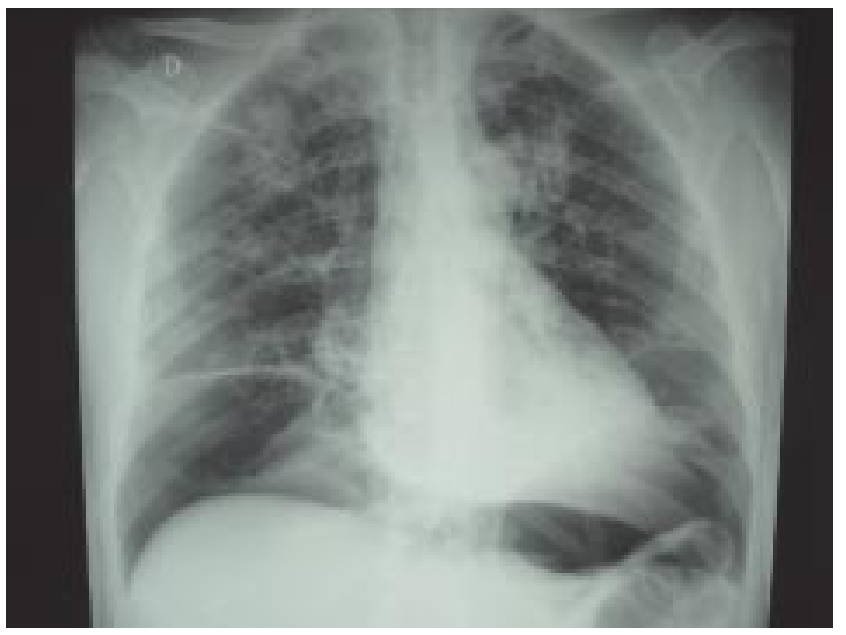

Figura 1 - Rx Tórax evidenciando o extenso pneumoperitonio. laparotomia exploradora para tratamento do pneumoperitonio, durante a qual foi constatada rotura em pequena curvatura gástrica de aproximadamente $7 \mathrm{~cm}$ de extensão; a conduta adotada foi rafia da lesão em dois planos e drenagem da cavidade. A paciente recebeu alta hospitalar no $14^{\circ}$ pós-operatório após término da antibióticoterapia e recuperação da insuficiência respiratória causada pela pneumonia.

\section{DISCUSSÃO}

As causas mais freqüentes de ruptura gástrica por barotrauma encontrados na literatura estão relacionadas a reanimação cardiopulmonar, oxigenoterapia com cateter nasal e acidentes com mergulhadores ${ }^{1,2,3}$.

A lesão gástrica por barotrauma pode apresentar-se sob diversas formas, desde lacerações parciais da mucosa até rotura completa da parede gástrica, devido à distensão crescente do órgão associada a espasmo do piloro e angulação do cárdia, o que impede a saída do ar, formando um mecanismo de válvula ${ }^{1}$.

A lesão gástrica ocorre na pequena curvatura em aproximadamente $73 \%$ dos $\operatorname{casos}^{1}$ e algumas séries de autópsias já revelaram a ocorrência de lacerações gástricas parciais em $10 \%$ dos pacientes submetidos a reanimação cardiopulmonar, todas na pequena curvatura ${ }^{4}$.

A ocorrência preferencial da lesão gástrica por barotrauma na pequena curvatura já foi atribuída exclusivamente à Lei de Laplace, segundo a qual a pressão dentro de uma cavidade é diretamente proporcional à tensão exercida em sua parede e inversamente proporcional ao raio desta cavidade, conforme a fórmula: $\mathrm{P}=\mathrm{T}\left(1 / \mathrm{R}_{1}+1 / \mathrm{R}_{2}\right)$.

Considerando que na pequena curvatura o raio na visão coronal está direcionado para fora e que na grande curvatura ambos direcionam-se para dentro, conclui-se que, na pequena curvatura, um dos raios tem sinal negativo. Portanto, neste caso, a soma dos raios será significativamente menor que na grande curvatura, exigindo assim maior tensão para resultar em um mesmo valor de $\mathrm{P}^{4}$.(Figura 2)

No entanto, esta teoria não considera a heterogenicidade da parede gástrica. A pequena curvatura freqüentemente apresenta largas pregas longitudinais em sua extensão com variações de espessura, sendo que as porções mais delgadas apresentam maior suscetibilidade aos aumentos de tensão ${ }^{5}$.

\footnotetext{
1. Profeesor Doutor do Departamento de Cirurgia e Ortopedia da Faculdade de Medicina de Botucatu.

2. Residente em Cirurgia do Aparelho Digestivo do Hospital das Clínicas da Faculdade de Medicina de Botucatu - UNESP.

Recebido em 13/06/2005

Aceito para publicação em 15/08/2005
}

Trabalho realizado no Departamento de Cirurgia e Ortopedia do Hospital das Clínicas da Faculdade de Medicina de Botucatu - UNESP. 


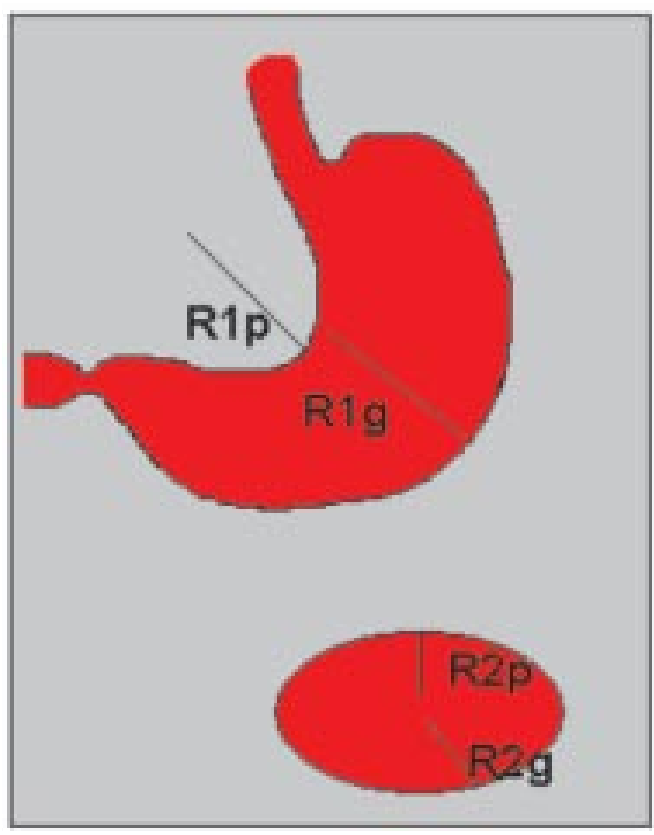

Figura 2 - Local preferencial de rotura na pequena curvatura, segundo a lei de Lapalce.
No presente caso optou-se pela intervenção cirúrgica devido ao estado séptico da paciente, a incerteza diagnóstica e a extensão do pneumoperitonio. Apesar de haver relatos na literatura ${ }^{1}$ de sucesso com o tratamento conservador, baseado em antibioticoterapia de largo espectro e drenagem gástrica via sonda com aspiração contínua, das rupturas gástricas por barotrauma, optamos pelo tratamento operatório em virtude do quadro clinico da paciente. Durante o procedimento verificamos que a rotura ocorreu na pequena curvatura, que é o local freqüentemente acometido ${ }^{1,4}$. Nossa opção pela ráfia em dois planos, decorreu das condições locais e da extensão da lesão conforme já relatado anteriormente. A causa provável da lesão gástrica desta paciente foi decorrente da ventilação com pressão positiva no esôfago.

Apesar da tuberculose e da pneumonia, a paciente apresentou boa evolução pós-operatória, não apresentando complicações.

Entendemos que todo procedimento realizado, como entubações e drenagens, deva ser seguido de checagem e cuidados imediatos para, assim, evitar complicações com esta.

\begin{abstract}
Barogenic rupture of the stomach is a rare complication following cardiopulmonary resuscitation, administration of nasal oxygen by catheter and diving accidents. We report a case of gastric barotrauma following oroesophageal intubation. In most cases, the tears occur along the lesser curvature, what have been already attributed to Laplace's formula and, more recently, to morphological features of the stomach (Rev. Col. Bras. Cir. 2007; 34(2): 138-139).
\end{abstract}

Key words: Stomach rupture; Intubation; Barotrauma

\title{
REFERÊNCIAS
}

1. Yeung P, Crowe P, Bennett M. Barogenic rupture of the stomach: a case for non-operative management. Aust N Z J Surg. 1998;68(1):76-7.

2. Cole DS, Burcher SK. Accidental pneumatic rupture of oesophagus and stomach. Lancet. 1961;1:24-5.

3. Darke SG, Bloomfield E. Case of complete gastric rupture complicating resuscitation. Br Med J. 1975;3(5980):414-5.

4. Barker SJ, Karagianes T. Gastric barotrauma: a case report and theoretical considerations. Anesth Analg. 1985;64(10):1026-8.

5. Kempen PM. Static and dynamic considerations in gastric barotrauma. Anesth Analg. 1986;65(5):540-1.
Como citar este artigo:

Saad-Hossne R, Prado RG, Bakoniy Neto A, Pereira RSC, Arashiro RTG. Rotura gástrica por barotrauma: relato de caso. Rev Col Bras Cir. [periódico na Internet] 2007; 34(2). Disponível em URL: http://www.scielo.br/rcbc

Endereço para correspondência:

Rogério Saad-Hossne

R. Tenente Silvio Besteti, 366 - Vila Padovan

Botucatu - SP

18607-690 